\title{
Conduction disturbances following surgical aortic valve replacement with a rapid-deployment bioprosthesis
}

Iuliana Coti, MD, ${ }^{\mathrm{a}}$ Christoph Schukro, MD, ${ }^{\mathrm{b}}$ Fitim Drevinja, MD, ${ }^{\mathrm{a}}$ Thomas Haberl, MD, ${ }^{\mathrm{a}}$ Alexandra Kaider, MD, ${ }^{\mathrm{c}}$ Alfred Kocher, $\mathrm{MD},{ }^{\mathrm{a}}$ Guenther Laufer, MD, ${ }^{\mathrm{a}}$ and Martin Andreas, MD, $\mathrm{PhD}^{\mathrm{a}}$

\section{ABSTRACT}

Background: The implantation of rapid-deployment aortic valves may interfere with the conduction system of the heart. This study evaluates the occurrence and predictive factors of conduction anomalies in patients undergoing surgical aortic valve replacement (SAVR) with a rapid-deployment balloon-expandable bioprosthesis in a single-center, real-world experience.

Methods: Between May 2010 and April 2019, 700 consecutive patients were included in a prospective, ongoing database with a longitudinal follow-up preoperatively, at discharge, and at 3 months, 1 year, 3 years, and 5 years postoperatively. Thirty-seven patients $(5.3 \%)$ had a permanent pacemaker at baseline and were excluded from further analysis, leaving 663 patients for analysis. Assessment of conduction anomalies was performed by electrocardiography (ECG) monitoring and repeated 12-lead ECG during the hospital stay and at postoperative follow-ups.

Results: Preoperatively, 126 patients (19.0\%) presented with different conduction disturbances. New permanent pacemaker implantation (PPI) occurred in 61 patients during the first 14 days (cumulative incidence, 9.4\%). The indication for PPI was complete atrioventricular block in 47 cases $(77 \%)$. Preoperative conduction anomalies, such as right bundle branch block, as well as operative characteristics (concomitant procedures) were found to be independent predictors for new PPI. One-year survival was $98 \%$ in patients with new early PPI and $96 \%$ in those without new early PPI $(P=.60)$.

Conclusions: The PPI rate was in the range of previous reports for rapiddeployment prostheses. PPI did not have a significant influence on short- to intermediate-term survival. Case selection with exclusion of patients presenting with baseline conduction disturbances may decrease the rate of new PPIs after SAVR with rapid-deployment balloon-expandable bioprostheses. (J Thorac Cardiovasc Surg 2021;162:803-11)

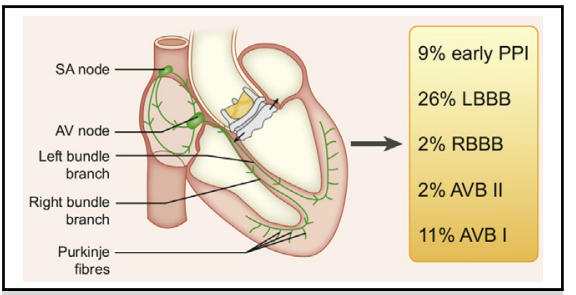

Conduction disturbances after RD-AVR: incidence and anatomic considerations.2

\section{CENTRAL MESSAGE}

The rapid-deployment Edwards

Intuity valve system has been

associated with an increased

occurrence of postoperative

conduction disturbances. Patient

selection may reduce the post-

operative pacemaker rate and

improve outcome.

\section{PERSPECTIVE}

Rapid-deployment aortic bioprostheses may interfere with the cardiac conduction system and lead to higher rates of postoperative conduction disturbances and permanent pacemaker implantation. Exclusion of patients with such baseline conduction anomalies as right bundle branch block may lead to a postoperative pacemaker implantation rate comparable to that with conventional sutured surgical prostheses.

See Commentaries on pages 812 and 813
Over the last decade, the development of rapid-deployment and sutureless aortic bioprostheses has led to major improvements in the surgical treatment of aortic stenosis. ${ }^{1} \mathrm{Ad}-$ vantages over conventional valves include reduced procedural time and better hemodynamics, along with the

From the ${ }^{\mathrm{a}}$ Division of Cardiac Surgery, Department of Surgery, ${ }^{\mathrm{b}}$ Division of Cardiology, Department of Internal Medicine II, and ${ }^{\mathrm{c} C e n t e r}$ for Medical Statistics, Informatics, and Intelligent Systems, Medical University of Vienna, Vienna, Austria.

Patients from multicenter clinical trials funded by Edwards Lifesciences (Irvine, Calif) were included in this analysis. Our institution receives financial support from the same company to conduct a long-term follow up after Intuity valve implantation.

Read at the 99th Annual Meeting of The American Association for Thoracic Surgery, Toronto, Ontario, Canada, May 4-7, 2019. facilitation of minimally invasive surgery owing to the ease of implantation. ${ }^{2-5}$ Rapid-deployment bioprostheses are inspired by transcatheter valve technologies, requiring balloon deployment of a subannular stainless steel frame, which may interfere with the cardiac conduction system

Received for publication July 11, 2019; revisions received Jan 12, 2020; accepted for publication Jan 19, 2020; available ahead of print Feb 19, 2020.

Address for reprints: Martin Andreas, MD, PhD, Medical University of Vienna, Waehringer Guertel 18-20, 1090 Vienna, Austria (E-mail: martin.andreas@ meduniwien.ac.at).

0022-5223

Copyright (C) 2020 The Author(s). Published by Elsevier Inc. on behalf of The American Association for Thoracic Surgery. This is an open access article under the CC BY-NC-ND license (http://creativecommons.org/licenses/by-nc-nd/4.0/). https://doi.org/10.1016/j.jtcvs.2020.01.083 


\section{Abbreviations and Acronyms \\ AVB = atrioventricular block \\ AVR = aortic valve replacement \\ $\mathrm{CABG}=$ coronary artery bypass grafting \\ $\mathrm{CI}=$ confidence interval \\ cSAVR $=$ combined surgical aortic valve replacement \\ ECG = electrocardiography \\ HR = hazard ratio \\ IQR = interquartile range \\ LAHB $=$ left anterior hemiblock \\ LBBB = left bundle branch block \\ $\mathrm{OR} \quad=$ odds ratio \\ PPI = permanent pacemaker implantation \\ $\mathrm{RD}-\mathrm{AVR}=$ Rapid-deployment aortic valve replacement \\ $\mathrm{RBBB}=$ right bundle branch block \\ SAVR = surgical aortic valve replacement \\ TAVR $=$ transcatheter aortic valve replacement}

$\checkmark$ Scanning this QR code will take you to the table of contents to access supplementary information. To view the AATS Annual Meeting Webcast, see the URL next to the webcast thumbnail.

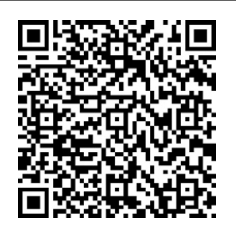

and may lead, as in transcatheter aortic valve replacement (TAVR), to a higher rate of permanent pacemaker implantation (PPI) compared with surgical aortic valve replacement (SAVR) with a conventional prosthesis. Early studies of AVR with the Edwards Intuity valve system (Edwards Lifesciences; Irvine, Calif) reported a postoperative PPI rate ranging from $5 \%$ to $12 \%,{ }^{6-9}$ higher than the $2 \%$ to $4 \%$ rate observed after AVR with conventional prostheses. ${ }^{10-12}$ Our institution participated in the market release trial of the Edwards Intuity valve system, which was standardized at our department with 700 implants over a 9-year period. The aims of this study were to comprehensively analyze the occurrence of postoperative conduction disturbances in patients undergoing rapid-deployment aortic valve replacement (RD-AVR), either in isolation or in combination with another procedure, and to define predictive risk factors that may lead to PPI in these patients.

\section{METHODS}

\section{Study Design}

All 700 consecutive patients who underwent isolated or combined RDAVR with the Edwards Intuity valve system between May 2010 and April 2019 at the Department of Cardiac Surgery at the Medical University of Vienna were included in a prospective, ongoing database with longitudinal endpoint assessment, as described in our previous report. ${ }^{8}$ In the present study, we analyzed all patients who received an RD-AVR either in isolation or with another concomitant procedure without a preexisting implanted pacemaker or implantable cardioverter-defibrillator. Thirty-seven patients $(5.3 \%)$ had a permanent pacemaker at baseline and were excluded from further analysis, leaving 663 patients for our analysis. Assessment of conduction anomalies was performed by electrocardiography (ECG) and repeated 12-lead ECGs during the hospital stay and at postoperative follow-ups. Clinical follow-up was performed preoperatively, at discharge, and at 3 months, 1 year, 3 years, and 5 years postoperatively. Assessment of conduction anomalies included ECG monitoring and repeated 12-lead ECGs during the hospital stay and at postoperative follow-ups. Between the clinical visits, telephone interview follow-ups were performed at 2 , 4 , and 7 years and updated before we closed the data analysis; each patient was interviewed regarding a new pacemaker implantation. When the event occurred in other institution, the medical records were requested and analyzed.

The university's Institutional Review Board approved this registry (1861/2016) and each patient provided written informed consent.

The total duration of follow-up was up to 9 years, with a median of 20 months (interquartile range [IQR], 11-41 months). The database was locked at the end of April 2019, when each patient reached at least 30 days of follow-up. The surgical procedure has been described in detail previously. ${ }^{6}$

\section{Study Endpoints}

The primary endpoint was the assessment of early PPI after RD-AVR. Early postoperative PPI was defined as occurring within 14 days after the index procedure, according to the current guidelines for reporting morbidity and mortality after cardiac valve operations. ${ }^{13}$ As secondary endpoints, we analyzed the overall occurrence of PPI with time 0 as the time of the index procedure, as well as the new onset of any atrioventricular or intraventricular conduction disturbance. The overall cumulative incidence of PPI was calculated, considering death as a competing event. Each PPI was documented; operative reports and the pre-PPI ECGs were examined for this analysis. Time to PPI was defined as the number of days between valve surgery and PPI. The indications for PPI were classified as complete atrioventricular block (AVB), intermittent third-degree AVB, Mobitz II AVB, or sick sinus syndrome. The timing of PPI did not follow a standard institutional protocol but instead was based mainly on the threshold of the temporary pacemaker or on the surgeon's judgment. Patients who underwent PPI and pacemaker interrogation at our institution were also analyzed for pacemaker dependency at follow-up. Pacemaker follow-up was performed at 1 year after implantation; 51 of 80 patients $(64 \%)$ were analyzed. Pacemaker dependency was defined as the absence of an intrinsic rhythm of $30 \mathrm{bpm}$ during backup pacing and after switching off the pacemaker and $>90 \%$ paced ventricular beats.

\section{Mortality}

For the calculation of overall mortality, we included all deaths after valve implantation regardless of cause. Early mortality was defined as mortality occurring during first 30 days post-PPI. Operative mortality was calculated as the cumulative value of early and in-hospital mortality, or mortality occurring at any other acute care facility to which a patient had been transferred following surgery.

\section{Statistical Analysis}

Descriptive statistical methods were applied to analyze preoperative and intraoperative characteristics and postoperative outcomes of the study population. Continuous variables were recorded as mean \pm standard deviation (SD) or median (IQR) for variables with a skewed distribution. Total numbers and proportions were reported for categorical outcomes. Statistical calculations comparing continuous variables were done using the $t$ test 
or the Wilcoxon Mann-Whitney test in cases of nonnormally distributed variables. Comparisons of categorical variables were done using Pearson's $\chi^{2}$ test. The median duration of follow-up time was estimated using the inverse Kaplan-Meier method. ${ }^{14}$

Competing-risk analysis was performed to estimate the cumulative incidence of PPI after RD-AVR, considering death as the competing event. A standard competing-risks method was used to estimate the cumulative incidence of PPI as described previously. ${ }^{15}$

To evaluate the possible influence of PPI on survival, a landmark analysis was performed considering day 14 after RD-AVR as the landmark. For this purpose, patients were grouped with respect to the occurrence or nonoccurrence of PPI up to day 14, and this landmark time point was considered the starting time point for the survival analysis. Thus, only patients with follow-up $>14$ days were included in this analysis. The Kaplan-Meier method was then performed to estimate survival probabilities, and the log-rank test was used for comparisons between groups. In addition, univariate and multivariable Cox regression models were performed to estimate the unadjusted and confounder-adjusted effects of PPI on survival. In addition to the risk factor PPI (up to day 14: yes vs no), the potential risk factors age, concomitant procedures, coronary artery bypass grafting $(\mathrm{CABG})$, and new onset of postoperative left bundle branch block (LBBB) were included in the multivariable regression model.

To identify possible baseline predictive factors for PPI within the first 14 days after surgery, we performed univariate and multivariable logistic regression analyses including the following variables: age, implanted valve size $(\mathrm{mm})$, concomitant procedures, concomitant CABG, and baseline conduction anomalies, such as right bundle branch block (RBBB), left-anterior hemiblock (LAHB), and first-degree AVB. Patients with a follow-up $<14$ days of follow-up $(n=39)$ and patients who died within the first 14 days after RD-AVR $(n=2)$, as well as patients with missing data on age or preoperative conduction anomalies RBBB, LAHB and first-degree AVB $(n=13)$ were not evaluable with respect to this endpoint; therefore, 609 patients were included in the multivariable logistic regression model. Univariate and multivariable Cox regression models were used, considering the outcome of PPI over the entire follow-up period as a secondary endpoint. The same potential risk factors as for the primary outcome of early PPI were included. All patients were evaluated in these regression models $(n=646)$, except for those with missing values $(\mathrm{n}=18)$.

Statistical analyses were performed using SPSS version 25 for Mac (IBM, Armonk, NY) and SAS version 9.4 (SAS Institute, Cary, NC). A $P$ value $<.05$ was considered to indicate statistical significance.

\section{RESULTS}

\section{Baseline Characteristics}

Between May 2010 and April 2019, a total of 700 patients underwent SAVR with the Edwards Intuity valve system at our institution. Among these patients, 37 (5.3\%) had a previous implanted pacemaker or implantable cardioverterdefibrillator and were excluded from the analysis. A total of 663 patients who underwent RD-AVR either in isolation or with another concomitant procedure were analyzed. The mean patient age was $73.4 \pm 7.8$ years (range, 29 to 92 years), and $300(45.2 \%)$ were women. Preoperatively, 126 patients $(19.0 \%)$ presented with 1 or more conduction disturbances: LBBB in $35(5.3 \%)$, RBBB in $38(5.7 \%)$, LAHB in $33(5.0 \%)$, first degree AVB in $37(5.6 \%)$, and second-degree AVB II in $3(0.5 \%)$. Atrial fibrillation was present in 122 patients $(18.4 \%), 70(10.6 \%)$ of whom had paroxysmal atrial fibrillation. The median predicted operative risk as estimated by EuroScore II and Society of Thoracic Surgeons score was 2.3 (IQR, 1.4-4.2) and 2.2 (IQR, 1.4-3.4), respectively. Preoperative characteristics and cardiovascular comorbidities are presented in Table 1.

\section{Operative Data}

Concomitant procedures were performed in 317 patients $(47.8 \%)$, including CABG in $207(31.2 \%)$, mitral valve surgery in $41(6.2 \%)$, and epicardial or full maze surgery in $39(5.9 \%)$; additional combined procedures are detailed in Table 2. The mean cardiopulmonary bypass and aortic cross-clamping times were $120 \pm 44$ minutes and $81 \pm 31$ minutes, respectively, in the total population and $105 \pm 30$ minutes and $70 \pm 23$ minutes, respectively, in patients undergoing isolated RD-AVR.

\section{PPI: Indication, Timing, Predictors}

A total of 61 patients underwent PPI in the first 14 days after the primary RD-AVR (Table 3). Over the entire follow-up period, 80 patients underwent PPI. Analysis of the cumulative incidence of PPI with death as the competing event revealed a PPI rate of $9.4 \%$ at 14 days, $10.3 \%$ at 30 days, $11.7 \%$ at 1 year and $15.3 \%$ at 5 years postsurgery (Figure 1). The pacing mode was synchronous (DDD) in 70 patients, single-chamber demand (VVI) in 8 patients and double-chamber CRT with pacemaker function in 2 patients. Overall, 56 patients $(70 \%)$ underwent PPI due to complete AVB, $4(5 \%)$ due to intermittent third-degree AVB, $5(6.3 \%)$ due to Mobitz II AVB, and the remainder due to symptomatic bradycardia (associated or not with atrial fibrillation). In patients without a baseline conduction disturbance, PPI in the first 14 postoperative days after RDAVR was required in 34 patients $(5.1 \%$ ), of whom $70.6 \%$ had a complete AVB. Of the PPI procedures performed within the first 14 days after the index procedure, the median timing was 7 days (IQR, 5-13.7 days). Among the 61 patients requiring PPI in the first 14 postoperative days, 2 $(3.3 \%)$ had concomitant mitral valve surgery, $4(6.6 \%)$ had tricuspid valve surgery, $4(6.6 \%)$ had septal myectomy, $6(9.8 \%)$ had surgical treatment of atrial fibrillation (either the maze procedure or pulmonary vein isolation only), and $12(19.7 \%)$ had preoperative atrial fibrillation.

The multivariable regression analysis of associations between baseline patient characteristics and operative data to all-cause PPI at 14 days revealed a significantly higher risk of postoperative PPI in patients with the preoperative conduction anomaly RBBB (odds ratio [OR], 9.85; 95\% confidence interval $[\mathrm{CI}], 4.43-21.89 ; P<.0001)$. RD-AVR performed concomitantly with other procedures was an independent predictor for elevated risk of PPI (OR, 5.82; $95 \%$ CI, 2.86-11.84; $P<.0001)$.

In univariate analysis, concomitant procedures alone, such as CABG, mitral/tricuspid valve surgery, or surgical treatment of atrial fibrillation, were not significant 
TABLE 1. Demographic characteristics

\begin{tabular}{|c|c|}
\hline Characteristic & Value \\
\hline Age, $y$, mean $\pm S D$ & $73.4 \pm 7.8$ \\
\hline Female sex, n (\%) & $300(45.2)$ \\
\hline Height, $\mathrm{cm}$, mean $\pm \mathrm{SD}$ & $169.4 \pm 9.3$ \\
\hline Weight, $\mathrm{kg}$, mean $\pm \mathrm{SD}$ & $80.8 \pm 16.1$ \\
\hline Body mass index, $\mathrm{kg} / \mathrm{m}^{2}$, mean $\pm \mathrm{SD}$ & $27.9 \pm 5.2$ \\
\hline Body surface area, $\mathrm{m}^{2}$, mean $\pm \mathrm{SD}$ & $1.92 \pm 0.21$ \\
\hline Logistic EuroSCORE, \%, median (IQR) & $6.6(4.0-11.6)$ \\
\hline EuroSCORE II, \%, median (IQR) & $2.3(1.4-4.2)$ \\
\hline STS score, $\%$, median (IQR) & $2.2(1.4-3.4)$ \\
\hline Diabetes, $\mathrm{n}(\%)$ & $182(28)$ \\
\hline Hypertension, n (\%) & $564(85)$ \\
\hline Coronary artery disease, $\mathrm{n}(\%)$ & $270(41)$ \\
\hline Cerebrovascular disease, $\mathrm{n}(\%)$ & $109(17)$ \\
\hline Renal insufficiency, n (\%) & $63(10)$ \\
\hline Creatinine, mean $\pm \mathrm{SD}$ & $1.08 \pm 0.72$ \\
\hline Peripheral vascular disease, n (\%) & $42(6)$ \\
\hline Chronic lung disease, $\mathrm{n}(\%)$ & $106(16)$ \\
\hline Previous cardiovascular interventions, n (\%) & $59(9)$ \\
\hline Previous valve surgery, $\mathrm{n}(\%)$ & $19(3)$ \\
\hline Previous conduction abnormalities, $\mathrm{n}(\%)$ & $126(19)$ \\
\hline Previous first-degree AVB, n (\%) & $37(6)$ \\
\hline Previous LBBB, n (\%) & $35(5)$ \\
\hline Previous RBBB, n (\%) & $38(6)$ \\
\hline Previous LAHB, n (\%) & $33(5)$ \\
\hline Bifascicular block & $12(2)$ \\
\hline Previous atrial fibrillation, $\mathrm{n}(\%)$ & $122(18)$ \\
\hline Paroxysmal AF, n (\%) & $70(11)$ \\
\hline Baseline PR interval, ms, mean \pm SD & $168 \pm 35$ \\
\hline Baseline QRS width, ms, mean $\pm \mathrm{SD}$ & $93 \pm 21$ \\
\hline
\end{tabular}

$S D$, Standard deviation; $I Q R$, interquartile range; STS, Society of Thoracic Surgeons; $A V B$, atrioventricular block; $\angle B B B$, left bundle branch block; $R B B B$, right bundle branch block; $L A H B$, left anterior hemiblock; $A F$, atrial fibrillation.

predictors of early PPI. However, patients with more than 2 concomitant procedures have shown a significantly higher risk of early PPI implantation.

All variables included in the univariate and multivariable analyses are listed in Table 4. When the overall PPI was analyzed (Table 5) with the index procedure as time 0 , not only preoperative RBBB (hazard ratio [HR], 4.86; 95\% CI, 2.74-8.63; $P<.001$ ) but also other preoperative conduction anomalies, including first-degree AVB (HR, 2.52; 95\% CI, $1.28-4 ; P=.117)$ and LAHB (HR, 2.06; 95\% CI, 1.054.02; $P=.035)$, were significant predictors for PPI in the multivariate Cox analysis. The mean postoperative hospital stay was longer for patients requiring a PPI $(16.2 \pm 17.3$ days vs $12.5 \pm 12.2$ days; $P=.028)$.
TABLE 2. Intraoperative characteristics

\begin{tabular}{lc}
\hline \multicolumn{1}{c}{ Characteristic } & Number $(\%)$ \\
\hline Access & \\
Full sternotomy & $350(53)$ \\
Hemi-sternotomy & $146(22)$ \\
Thoracotomy & $167(25)$ \\
Concomitant procedures & $318(48)$ \\
CABG & $208(31)$ \\
Aortic reduction plasty & $42(6)$ \\
Atrial fibrillation surgery (endo/epicardial ablation) & $39(6)$ \\
MVR/MVr & $28(4)$ \\
TVr & $28(4)$ \\
Aortic valve anatomy & \\
Tricuspid & $565(85)$ \\
Bicuspid & $83(13)$ \\
Previous prosthesis & $15(2)$ \\
Implanted valve size & \\
19 mm & $69(10.4)$ \\
$21 \mathrm{~mm}$ & $158(23.8)$ \\
$23 \mathrm{~mm}$ & $214(32.3)$ \\
$25 \mathrm{~mm}$ & $155(23.4)$ \\
$27 \mathrm{~mm}$ & $67(10.1)$ \\
\hline$C A B G$, Coronary artery bypass grafting; $M V R$, mitral valve replacement; $M V r$, mitral \\
valve reconstruction; $T V r$, tricuspid valve reconstruction.
\end{tabular}

\section{New Onset of Postoperative Bundle Branch Block}

New onset of LBBB before discharge occurred in 206 patients $(31.1 \%)$; it was transient in 36 of these patients $(5.4 \%)$, with complete regression at discharge, whereas 170 patients $(25.6 \%)$ had LBBB at discharge. Incomplete LBBB or new occurrence of LAHB was present in 18 patients $(2.7 \%)$ before discharge. New onset of RBBB occurred in 11 patients $(1.7 \%)$ before discharge. Longer baseline QRS width and PR interval were associated with a higher incidence of new-onset LBBB $(P<.001)$. A QRS duration $>150 \mathrm{~ms}$ was found in 127 patients $(61.7 \%)$ with new-onset LBBB (Table 3).

\section{Pacemaker Dependency After PPI}

Fifty-one of 80 patients $(64 \%)$ completed 1 year of follow-up and/or had a pacemaker check at our institution. Among these patients, $31(60.1 \%)$ were pacemakerdependent, defined as absence of an intrinsic rhythm of 30 bpm during back-up pacing and after switching off the pacemaker and $>90 \%$ paced ventricular beats. All of these patients had undergone PPI due to complete AVB.

\section{Mortality}

Four patients died during the first 30 days after PPI $(0.6 \%)$. Eight $(1.2 \%)$ operative deaths occurred, all in patients with concomitant procedures. Landmark survival analysis revealed survival after early PPI of $98 \%$ at 1 year, $86 \%$ at 3 years, and $82 \%$ at 5 years after surgery; PPI did not have a significant impact on survival 
TABLE 3. In-hospital postoperative conduction anomalies

\begin{tabular}{lc}
\hline \multicolumn{1}{c}{ Factor } & Value \\
\hline New-onset postoperative conduction anomalies, n $(\%)^{*}$ & $310(47)$ \\
First-degree AVB & $72(11)$ \\
LAHB & $18(3)$ \\
LBBB & $206(31)$ \\
Transient LBBB & $36(5)$ \\
RBBB & $11(2)$ \\
Second-degree AVB & $10(2)$ \\
Complete AVB & $59(9)$ \\
PPI $\dagger$ & \\
$\leq 14$ d & $61(9)$ \\
$\leq 30$ d & $68(10)$ \\
PR interval predischarge, $m s$, mean \pm SD & $178 \pm 43$ \\
QRS width predischarge, $m s$, mean \pm SD & $115 \pm 30$
\end{tabular}

$\overline{A V B}$, Atrioventricular block; $\angle A H B$, left anterior hemiblock; $L B B B$, left bundle branch block; $R B B B$, right bundle branch block; $P P I$, permanent pacemaker implantation; $S D$, standard deviation. *Overall postoperative new-onset of conduction anomalies following RD-AVR. †PPI incidence was analyzed by means of cumulative incidence, with mortality as competing event (percentage provided according to this analysis).

(Figure 2). Univariate and multivariable Cox regression models were performed to estimate the unadjusted and confounder-adjusted effect of PPI on survival. In the univariate model, age (HR, 1.05; 95\% CI, 1.01-1.08; $P=.017)$, concomitant procedures (HR, 2.38, 95\% CI, 1.39-4.07;
$P=.002)$, and concomitant CABG (HR, 2.12; 95\% CI, $1.28-3.52 ; P=.004)$ were predictive of mortality. In the multivariate analysis, only concomitant procedures (including multiple procedures besides the AVR) and advanced age remained significant predictors of mortality.

\section{DISCUSSION}

The Edwards Intuity valve system combines a rapidly deployable stent-based subannular fixation system with an established biological valve prosthesis. The subannular stent frame exerts compression on the conduction system and, similar to various transcatheter aortic valves, might exert trauma to the conduction system when the balloonexpandable subannular stent is deployed, owing to the anatomic proximity to the bundle of His (Figure 3). Moreover, debridement of a massively calcified annulus in the region of the membranous septum beneath the commissure separating the noncoronary and right coronary cusp may also injure the bundle of His (Video 1).

Different baseline characteristics, including age, bundle branch block, or and first-degree AVB, were integrated into a multivariable logistic regression model to identify factors predictive of PPI after RD-AVR. The presence of a baseline conduction disturbance, such as RBBB, was found to be an independent predictor for PPI. Other factors, such as advanced age and concomitant procedures, were also

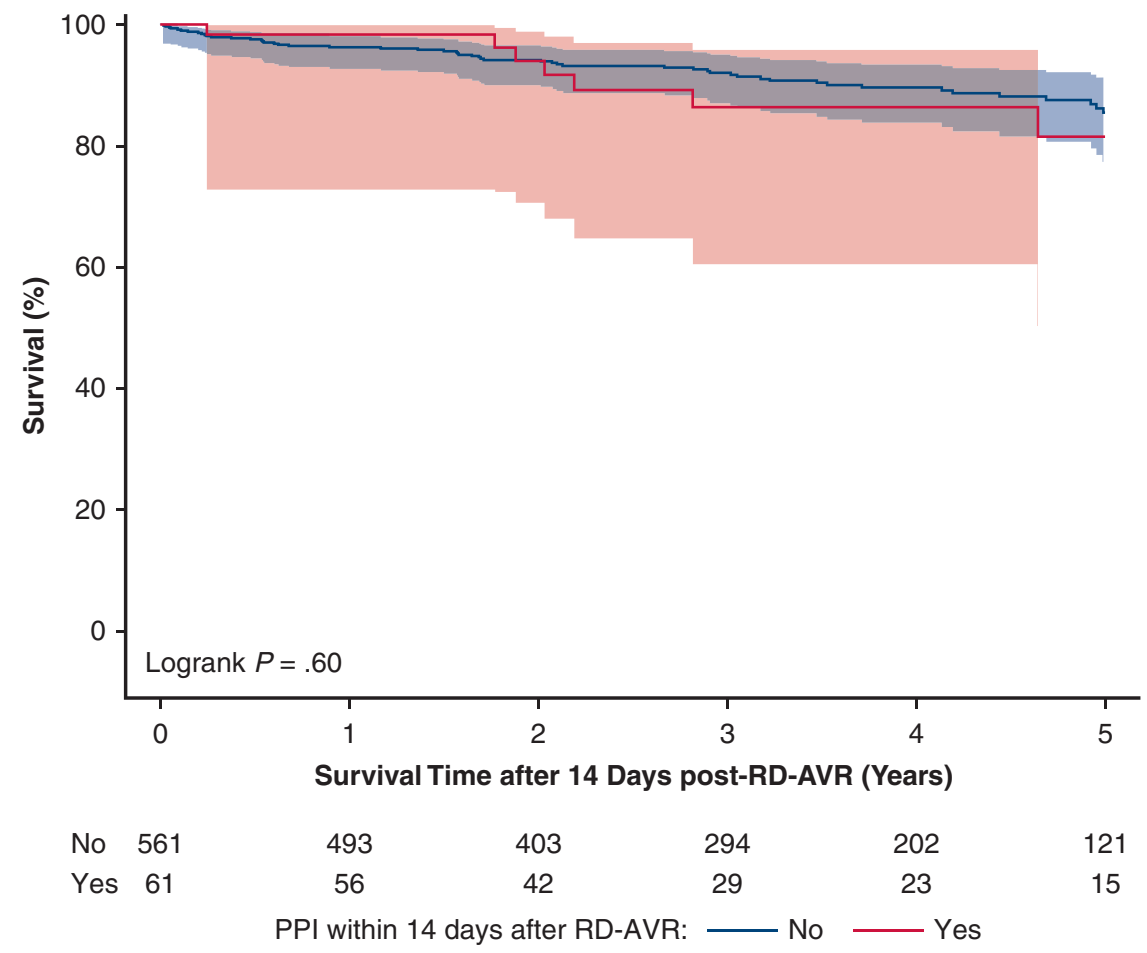

FIGURE 1. Landmark analysis: overall survival at 14 days after rapid-deployment aortic valve replacement $(R D-A V R)$. Kaplan-Meier survival-analysis, log-rank test. Blue, pacemaker group; red, no pacemaker group. Survival was similar in patients requiring a PPI in the first 14 days after RD-AVR and those who did not $(P=.60$, log-rank test). Only patients with duration of follow-up $>14$ days were included $(\mathrm{n}=622)$. $P P I$, Pacemaker implantation. 
TABLE 4. Univariate and multivariable logistic regression for early PPI predictors (first 14 days after RD-AVR)

\begin{tabular}{|c|c|c|c|c|c|}
\hline \multirow[b]{2}{*}{ Variable* } & \multirow[b]{2}{*}{ Events, $\mathbf{n}(\%)$} & \multicolumn{2}{|c|}{ Univariate analyses } & \multicolumn{2}{|c|}{ Multivariable analysis } \\
\hline & & OR $(95 \%$ CI $)$ & $P$ value & OR $(95 \%$ CI $)$ & $P$ value \\
\hline Age, y & & $1.06(1.02-1.10)$ & .006 & $1.08(1.03-1.13)$ & .001 \\
\hline \multicolumn{6}{|l|}{ Baseline } \\
\hline RBBB & $37(6)$ & $9.16(4.46-18.81)$ & $<.001$ & $9.85(4.43-21.89)$ & $<.001$ \\
\hline LAHB & $33(5)$ & $2.66(1.10-6.43)$ & .029 & $1.35(0.48-3.80)$ & .566 \\
\hline First-degree AVB & $37(6)$ & $2.29(0.96-5.46)$ & .062 & $2.19(0.82-5.82)$ & .117 \\
\hline Concomitant procedures & $295(47)$ & $2.11(1.22-3.66)$ & .008 & $5.82(2.86-11.84)$ & $<.001$ \\
\hline CABG & $192(31)$ & $0.78(0.43-1.41)$ & .410 & $0.17(0.08-0.38)$ & $<.001$ \\
\hline Bioprosthesis size (unit: $2 \mathrm{~mm}$ ) & & $1.20(0.95-1.52)$ & .124 & $1.26(0.97-1.63)$ & .087 \\
\hline
\end{tabular}

$O R$, Odds ratio; $C I$, confidence interval; $R B B B$, right bundle branch block; $L A H B$, left anterior hemiblock; $A V B$, atrioventricular block; $C A B G$, coronary artery bypass grafting. *Patients with a follow-up $<14$ days $(\mathrm{n}=39)$ and patients who died $(\mathrm{n}=2)$ within the first 14 days after RD-AVR, as well as patients with missing data regarding age or preoperative conduction anomalies_-RBBB, LAHB, or first-degree AVB $(n=13)$-were not evaluable with respect to this endpoint. Only 609 patients were included in the multivariable logistic regression model.

found to be independent predictors for PPI in the multivariable analysis. Erdogan and colleagues ${ }^{16}$ reported that bicuspid aortic valve, female sex, presence of RBBB and LBBB at baseline, prolonged perfusion time, and hypertension predicted an elevated risk of PPI after AVR with conventional aortic prostheses. Koplan and colleagues ${ }^{17}$ identified preoperative RBBB, LBBB, preoperative PR interval $>200 \mathrm{~ms}$, concomitant procedures, and older age as predictive of postoperative PPI. In the same study, preoperative RBBB was associated with a higher risk of PPI compared with LBBB.

Numerous studies have analyzed the occurrence of conduction anomalies after TAVR and the need for PPI in these patients, but less is known about this problem after SAVR with RD-AVR. Rahmanian and colleagues ${ }^{18}$ analyzed 193 patients undergoing RD-AVR and in a multivariate analysis identified preoperative RBBB and bypass grafting of the circumflex artery as independent predictors of PPI.

Romano and colleagues ${ }^{9}$ analyzed the occurrence of PPI and other conduction disturbances in 708 patients following RD-AVR, performed either in isolation or with concomitant CABG, previously enrolled in the North American
TRANSFORM trial. They found a rate of all-cause PPI to discharge of $12.3 \%$, higher than our finding of $9.5 \%$. In patients without any baseline conduction anomalies, the overall PPI rate predischarge was $6.6 \%$, also slightly higher than our $5.1 \%$. The mean time from RD-AVR to PPI was $5.2 \pm 3.5$ days (median, 5 days; IQR, 3-6 days), with $22 \%$ patients undergoing PPI within 2 days and $46 \%$ doing so within 4 days. In contrast to that study, $10 \%$ of our patients underwent PPI within the first 2 days and $25 \%$ did so in the first 4 days, with a median predischarge timing of 7 days (IQR, 5-13.7 days) in our entire cohort. As in other European centers, we prefer a longer observational period and leave the temporary pacemaker wires in place for at least 5 days after the index procedure, allowing the regression of possible transient conduction disturbances or bradyarrhythmias.

In the TRANSFORM trial, new onset of LBBB and RBBB occurred in $26 \%$ and $4 \%$ of patients, respectively, similar to our findings $31 \%$ and $2 \%$, respectively. Importantly, in our cohort, $5.4 \%$ of the new LBBBs were transient, and the patients recovered before discharge, with $26 \%$ patients showing LBBB at discharge, similar to the American cohort. As in the TRANSFORM trial, preoperative RBBB was

TABLE 5. Univariate and multivariable Cox regression analysis for predictors of PPI during the follow-up period

\begin{tabular}{|c|c|c|c|c|}
\hline \multirow[b]{2}{*}{ Prognostic factor* } & \multicolumn{2}{|c|}{ Univariate analyses } & \multicolumn{2}{|c|}{ Multivariable analysis } \\
\hline & HR $(95 \%$ CI) & $P$ value & HR $(95 \%$ CI) & $P$ value \\
\hline Age, $y$ & $1.06(1.03-1.09)$ & $<.001$ & $1.06(1.03-1.10)$ & $<.001$ \\
\hline \multicolumn{5}{|l|}{ Baseline } \\
\hline RBBB & $5.84(3.40-10.03)$ & $<.001$ & $4.86(2.74-8.63)$ & $<.001$ \\
\hline LAHB & $3.41(1.80-6.46)$ & $<.001$ & $2.06(1.05-4.02)$ & .035 \\
\hline AVB I & $2.74(1.41-5.33)$ & .003 & $2.52(1.28-4.96)$ & .007 \\
\hline Concomitant procedures & $1.96(1.24-3.08)$ & .004 & $3.49(2.01-6.07)$ & $<.001$ \\
\hline CABG & $1.02(0.64-1.64)$ & .920 & $0.34(0.19-0.60)$ & $<.001$ \\
\hline Bioprosthesis size (unit: $2 \mathrm{~mm}$ ) & $1.17(0.97-1.42)$ & .110 & $1.12(0.92-1.37)$ & .260 \\
\hline
\end{tabular}

$\overline{H R}$, Hazard ratio; $C I$, confidence interval; $R B B B$, right bundle branch block; $L A H B$, left anterior hemiblock; $A V B I$, first-degree atrioventricular block; $C A B G$, coronary artery bypass grafting. *Patients with missing data on age or preoperative conduction anomalies-RBBB, LAHB, or AVB I- $(\mathrm{n}=15)$ were not evaluable with respect to this endpoint. Only 646 patients were included in the multivariable Cox regression model. 

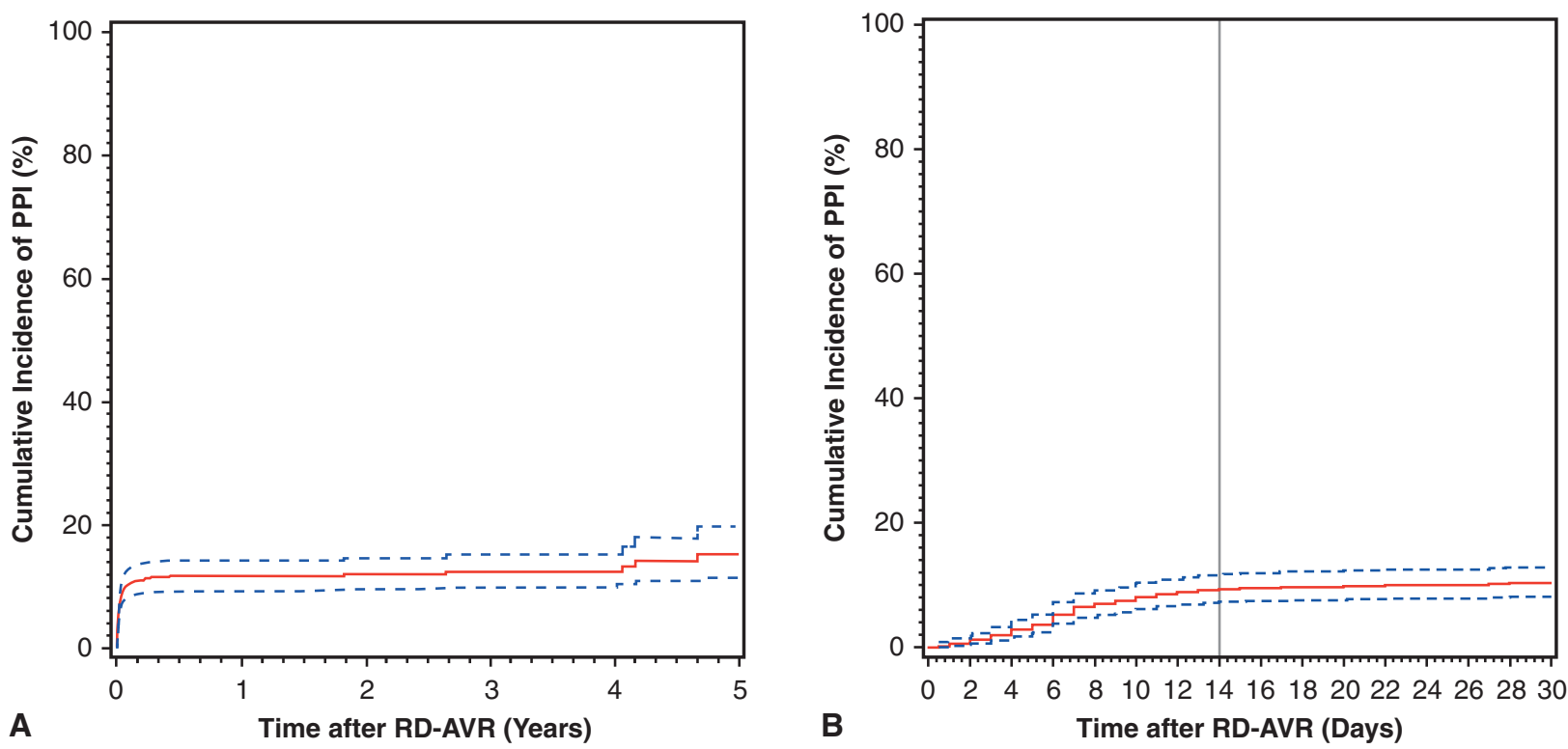

FIGURE 2. A, Cumulative incidence of permanent pacemaker implantation (PPI). Competing-risk analysis was performed to estimate the cumulative incidence of PPI after rapid-deployment aortic valve replacement $(R D-A V R)$ considering death as a competing event. $\mathrm{B}$, Cumulative incidence of permanent pacemaker implantation $(P P I)$ over the first 30 postoperative days. Competing-risk analysis was performed to estimate the cumulative incidence of PPI after RD-AVR considering death as competing event.

identified as an independent predictor for PPI following RD-AVR.

The other available sutureless valve (Perceval Sutureless Aortic Heart Valve; LivaNova, London, United Kingdom) is also associated with higher rates of postoperative conduction anomalies. Importantly, the risk of PPI is reduced over time in expert centers, indicating a correlation with implantation technique. ${ }^{1}$ A recent analysis from Lam and colleagues ${ }^{19}$ comparing the incidences of LBBB and PPI after sutureless and conventional AVR and the impact on survival revealed higher incidences of LBBB and PPI in the sutureless group $(16.7 \%$ vs $2.3 \%[P<.001]$ for new-onset $\mathrm{LBBB}$ and $6.8 \%$ vs $1.6 \%[P=.001]$ for PPI), but neither new-onset LBBB nor PPI was associated with increased mortality $(P=.20)$.

Van der Boon and colleagues ${ }^{20}$ reviewed several reports analyzing the incidence of conduction anomalies after TAVR with the 2 most used transcatheter technologies. The PPI rate varied from $18 \%$ to $49 \%$ after implantation of the self-expanding Medtronic CoreValve (Medtronic,

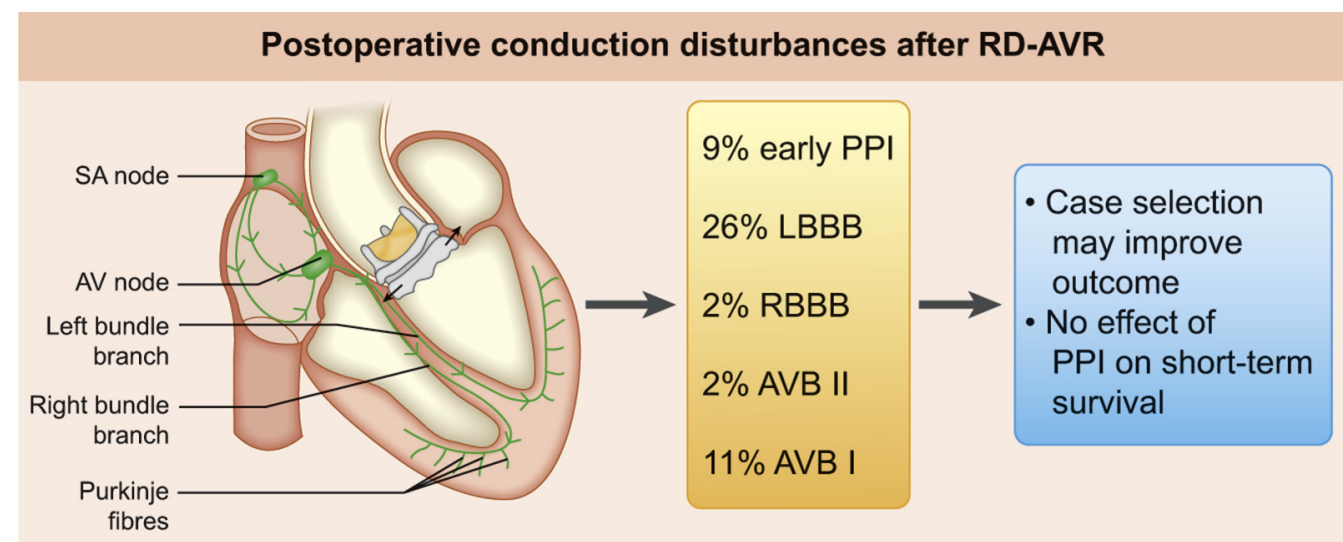

FIGURE 3. Conduction disturbances after rapid-deployment aortic valve replacement ( $R D-A V R)$ : incidence and anatomic considerations. The expansion of the subannular stent within the left ventricular outflow tract in the proximity of the left bundle branch (black arrows) may exert compression on the conduction system and influence the occurrence of postoperative conduction disturbances. $S A$, Sinoatrial; $A V$, atrioventricular; $P P I$, permanent pacemaker implantation; $L B B B$, left bundle branch block; $R B B B$, right bundle branch block; $A V B I$, first-degree atrioventricular block; $A V B I I$, second-degree atrioventricular block. 


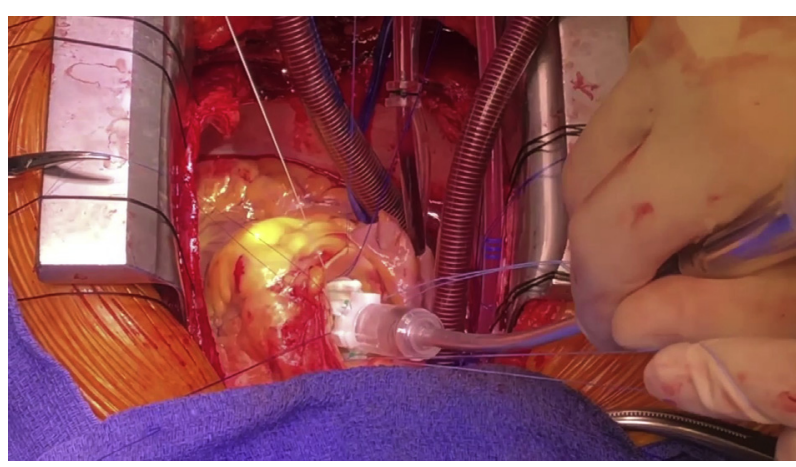

VIDEO 1. Conduction disturbances after surgical aortic valve replacement with the Edwards Intuity valve system: technical and anatomic aspects. Video available at: https://www.jtcvs.org/article/S0022-5223(20) 30433-5/fulltext.

Minneapolis, Minn) and from $0 \%$ to $12 \%$ after implantation of the balloon-expandable Edwards Sapien valve, and new onset of LBBB was $29 \%$ to $65 \%$ with the CoreValve and $4 \%$ to $18 \%$ with the Sapien valve. ${ }^{20}$ Temporary rhythm disturbances are common after cardiac surgery, especially after valve surgery, but may reverse during hospitalization. In our cohort, postoperative LBBB was transitory in $18 \%$ of the patients with new-onset complete LBBB until discharge. Chamandi and colleagues ${ }^{21}$ recently reported long-term outcomes in patients with new-onset persistent LBBB following TAVR. In their cohort, with a median follow-up of 3 years, persistent LBBB was not associated with higher mortality or rate of rehospitalization for heart failure, but was associated with an increased risk of PPI, especially in the first 12 months after TAVR, and with a negative impact on left ventricular function over time. In a recent meta-analysis of 41 studies including 11,210 TAVR recipients, Siontis and colleagues ${ }^{22}$ identified male sex, first-degree AVB, LAHB, and RBBB as preprocedural predictors of PPI. Our regression analysis identified RBBB as a significant predictor for PPI in the multivariable analysis, whereas LAHB remained a significant predictor for PPI only in the univariate analysis.

TAVR is widely accepted as a standard approach in intermediate and high-risk patients who are ineligible for standard cardiac surgery. The PARTNER 3 trial showed that in low-risk patients, TAVR was superior to SAVR in terms of reducing the risks of death, stroke, and rehospitalization at 1 year postprocedure ${ }^{23}$; nonetheless, the routine use of TAVR in lower-risk patient populations remains controversial, and long-term data are needed. A transcatheter valve displaces the native valve and anchors into the calcified area, whereas SAVR assumes complete decalcification before implantation, which may contribute to a higher incidence of paravalvular regurgitation and an elevated risk of subclinical leaflet thrombosis in TAVR recipients. ${ }^{24}$ In the absence of long-term follow-up data on TAVR in low-risk patients, it is too soon to anticipate that SAVR will be completely replaced by TAVR in these cases. Moreover, some patients require combined procedures with multiple valve pathologies or coronary artery disease and coronary lesions ineligible for PPI in whom CABG is indicated. Unfavorable anatomy, peripheral vascular disease, and bicuspid aortic valve may also represent contraindications for TAVR. The Edwards Intuity valve has shown excellent results concerning hemodynamic performance and safety, with the proven durability of Edwards Magna valve. This valve also facilitates minimally invasive approaches and may be beneficial in complex combined procedures.

In conclusion, the Edwards Intuity valve has been associated with a higher incidence of postoperative conduction disturbances compared with conventional valves. Nevertheless, almost one-half of our patients (44\%) requiring a PPI in the first 14 days after RD-AVR presented with different baseline conduction anomalies, of which RBBB was an independent predictor for a higher rate of PPI. PPI or new-onset LBBB did not have a significant influence on the excellent survival rate on early- and intermediate-term follow-up. Continued refinements in surgical technique and patient selection may further improve outcomes in these patients.

\section{Study Limitations}

This study is a cohort analysis that combines the results of premarket clinical trials and postmarket registries (FOUNDATION and MISSION studies, as well as our institutional registry which included all other patients receiving the Edwards Intuity Valve). All consecutive patients were included, which might have resulted in an inhomogeneous patient population owing to varying inclusion criteria over time.

\section{Webcast}

You can watch a Webcast of this AATS meeting presentation by going to: https://aats.blob.core.windows.net/media/ 19\%20AM/Monday_May6/206BD/206BD/S69\%20-\%20 Rapid\%20Fire \%20abstracts\%20IV\%20-\%20Valve/S69_ 9_webcast_081515404.mp4.

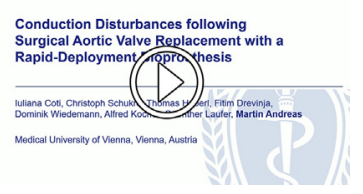

\section{Conflict of Interest Statement}

Dr Kocher has received speaking honoraria from Edwards Lifesciences. Dr Haberl has received research funding from Edwards Lifesciences. Dr Laufer has received consulting fees from Edwards Lifesciences. Dr Andreas has received research funding and speaking honoraria from 
Edwards Lifesciences, and has served as a proctor for Edwards Lifesciences and Abbott Laboratories and as an advisor for Medtronic.

\section{References}

1. Di Eusanio M, Phan K, Berretta P, Carrel TP, Andreas M, Santarpino G, et al. Sutureless and rapid-deployment aortic valve replacement international registry (SURD-IR): early results from 3343 patients. Eur J Cardiothorac Surg. 2018; 54:768-73.

2. Berretta P, Andreas M, Carrel TP, Solinas M, Teoh K, Fischlein T, et al. Minimally invasive aortic valve replacement with sutureless and rapid deployment valves: a report from an international registry (sutureless and rapid deployment international registry). Eur J Cardiothorac Surg. 2019;56:793-9.

3. Andreas M, Wallner S, Habertheuer A, Rath C, Schauperl M, Binder T, et al. Conventional versus rapid-deployment aortic valve replacement: a singlecentre comparison between the Edwards Magna valve and its rapiddeployment successor. Interact Cardiovasc Thorac Surg. 2016;22:799-805.

4. Borger MA, Dohmen PM, Knosalla C, Hammerschmidt R, Merk DR, Richter M, et al. Haemodynamic benefits of rapid deployment aortic valve replacement via a minimally invasive approach: 1-year results of a prospective multicentre randomized controlled trial. Eur J Cardiothorac Surg. 2016;50:713-20.

5. Capelli C, Corsini C, Biscarini D, Ruffini F, Migliavacca F, Kocher A, et al. Pledget-armed sutures affect the haemodynamic performance of biologic aortic valve substitutes: a preliminary experimental and computational study. Cardiovasc Eng Technol. 2017;8:17-29.

6. Kocher AA, Laufer G, Haverich A, Shrestha M, Walther T, Misfeld M, et al. Oneyear outcomes of the surgical treatment of aortic stenosis with a next generation surgical aortic valve (TRITON) trial: a prospective multicenter study of rapiddeployment aortic valve replacement with the EDWARDS INTUITY valve system. J Thorac Cardiovasc Surg. 2013;145:110-5; discussion: 115-6.

7. Laufer G, Haverich A, Andreas M, Mohr FW, Walther T, Shrestha M, et al. Longterm outcomes of a rapid deployment aortic valve: data up to 5 years. Eur J Cardiothorac Surg. 2017:52:281-7.

8. Andreas M, Coti I, Rosenhek R, Shabanian S, Mahr S, Uyanik-Uenal K, et al. Intermediate-term outcome of 500 consecutive rapid-deployment surgical aortic valve procedures. Eur J Cardiothorac Surg. 2019;55:527-33.

9. Romano MA, Koeckert M, Mumtaz MA, Slachman FN, Patel HJ, Chitwood WR Jr, et al. Permanent pacemaker implantation after rapid deployment aortic valve replacement. Ann Thorac Surg. 2018;106:685-90.

10. Van Mieghem NM, Head SJ, de Jong W, van Domburg RT, Serruys PW, de Jaegere PP, et al. Persistent annual permanent pacemaker implantation rate after surgical aortic valve replacement in patients with severe aortic stenosis. Ann Thorac Surg. 2012;94:1143-9.

11. Bagur R, Manazzoni JM, Dumont É, Doyle D, Perron J, Dagenais F, et al. Permanent pacemaker implantation following isolated aortic valve replacement in a large cohort of elderly patients with severe aortic stenosis. Heart. 2011;97: 1687-94.

12. Limongelli G, Ducceschi V, D’Andrea A, Renzulli A, Sarubbi B, De Feo M, et al Risk factors for pacemaker implantation following aortic valve replacement: a single centre experience. Heart. 2003;89:901-4.

13. Akins CW, Miller DC, Turina MI, Kouchoukos NT, Blackstone EH, Grunkemeier GL, et al. Guidelines for reporting mortality and morbidity after cardiac valve interventions. J Thorac Cardiovasc Surg. 2008;135:732-8.

14. Schemper M, Smith TL. A note on quantifying follow-up in studies of failure time. Control Clin Trials. 1996;17:343-6.

15. Kim HT. Cumulative incidence in competing risks data and competing risks regression analysis. Clin Cancer Res. 2007;13(2 Pt 1):559-65.

16. Erdogan HB, Kayalar N, Ardal H, Omeroglu SN, Kirali K, Guler M, et al. Risk factors for requirement of permanent pacemaker implantation after aortic valve replacement. J Card Surg. 2006;21:211-5; discussion: 216-7.

17. Koplan BA, Stevenson WG, Epstein LM, Aranki SF, Maisel WH. Developmen and validation of a simple risk score to predict the need for permanent pacing af ter cardiac valve surgery. J Am Coll Cardiol. 2003;41:795-801.

18. Rahmanian PB, Eghbalzadeh K, Kaya S, Menghesha H, Gerfer S, Liakopoulos OJ, et al. Determination of risk factors for pacemaker requirement following rapid-deployment aortic valve replacement. Interact Cardiovasc Thorac Surg. 2018;27:215-21.

19. Lam KY, Akca F, Verberkmoes NJ, van Dijk C, Claessens A, Soliman Hamad MA, et al. Conduction disorders and impact on survival after sutureless aortic valve replacement compared to conventional stented bioprostheses. Eur J Cardiothorac Surg. 2019;55:1168-73.

20. van der Boon RM, Nuis RJ, Van Mieghem NM, Jordaens L, Rodés-Cabau J, van Domburg RT, et al. New conduction abnormalities after TAVI-frequency and causes. Nat Rev Cardiol. 2012;9:454-63.

21. Chamandi C, Barbanti M, Munoz-Garcia A, Latib A, Nombela-Franco L, Gutiérrez-Ibanez E, et al. Long-term outcomes in patients with new-onset persistent left bundle branch block following TAVR. JACC Cardiovasc Interv. 2019;12: 1175-84.

22. Siontis GC, Jüni P, Pilgrim T, Stortecky S, Büllesfeld L, Meier B, et al. Predictors of permanent pacemaker implantation in patients with severe aortic stenosis undergoing TAVR: a meta-analysis. J Am Coll Cardiol. 2014;64:129-40.

23. Mack MJ, Leon MB, Thourani VH, Makkar R, Kodali SK, Russo M, et al. Transcatheter aortic-valve replacement with a balloon-expandable valve in low-risk patients. N Engl J Med. 2019;380:1695-705.

24. Hatoum H, Moore BL, Maureira P, Dollery J, Crestanello JA, Dasi LP. Aortic sinus flow stasis likely in valve-in-valve transcatheter aortic valve implantation. $J$ Thorac Cardiovasc Surg. 2017;154:32-43.e1.

Key Words: permanent pacemaker implantation, conduction disturbances, aortic valve replacement, rapid deployment 